\title{
Public-opened Internet Electron Microscopy 2005 in Japan ---- Remote-control SEM and TEM for High-school Users ----
}

\author{
K. Furuya, M. Tanaka, K. Mitsuishi, N. Ishikawa, A. Tameike, M. Date ${ }^{1}$, A. Yamada ${ }^{1}$ and Y. Okura $^{1}$ \\ National Institute for Materials Science, Tukuba 305-0003, Japan \\ ${ }^{1}$ JEOL Ltd., Akishima, Tokyo 196-8558, Japan
}

Public-opened Internet Electron Microscopy (POIT-EM) has successfully been developed in National Institute for Materials Science (NIMS), Tsukuba, Japan. Real-time observation and analysis of various samples can be performed from anyplace in the world (Fig. 1). This system uses data encryption and security mechanisms to ensure that only authorized users can access to the POIT-EM network. The client computers run on Windows XP. All kinds of operations necessary for the remote control of the SEM and TEM can be performed through the Internet; turning-on high voltage and changing magnification, focus, brightness and contrast. Previous systems based on a similar concept have been constructed for research purposes on ultra-high voltage microscopes ${ }^{1-4}$. Unlike these systems, POIT-EM system is not only for researchers but also for educational purposes for young people.

First version of the POIT-EM was developed in 2001 and preliminary run at National Museum of Emerging Science and Innovation (MIRAIKAN) at Daiba in Tokyo. Our statistic data revealed that more than half of the users, out of over 1,000 users, were children and students under 20 years old. This implies that POIT-EM is quite unique for science education. We have successfully installed the latest version of the POIT-EM client at seven high schools in 2004. For the general use at science classes and clubs, students send SEM samples to NIMS, which are set in the SEM by the technicians. Once SEM session is set up and opened, the students observe their samples by the client PCs, from their class rooms (Fig. 2).

Last year, we improved the network system to cope with various network environments of the users. This system employs a virtual private network (VPN), which encrypts user authentication information. The user can be identified by the pocket secure-ID card. And then, any PC can be used for remote operation if the proper software was already installed. We have carried out connection tests of the system through NIMS's intranet LAN, ADSL private lines (analogue lines) and optical fiber cables. The remote control of the microscopes with the different networks was confirmed, though the data transfer rate varies depending on the client-side bandwidth.

As the number of our POIT-EM system user increases, the management of users database becomes important. We are currently developing a multi-users login system which consists of two parts as shown in Fig. 3. The first one is a database which contains "users' ID", "sample position", "observation conditions" and "SEM session start/close time and date". The second one is a web-based groupware for checking/making reservations and for reporting current user's status such as the project name and information. The detail of the latest system will be described in the presentation.

References

[1] N. J Zaluzec , Teleconference Mag., 17 (1998)

[2] N. Yamada et al., Society of Neuroscience, 25 (1999)

[3] K. Yoshida et al., J.Electron Microsc., 48 (1999) 865 
[4] A. Takaoka et al., Ultramicroscopy, 83 (2000) 93
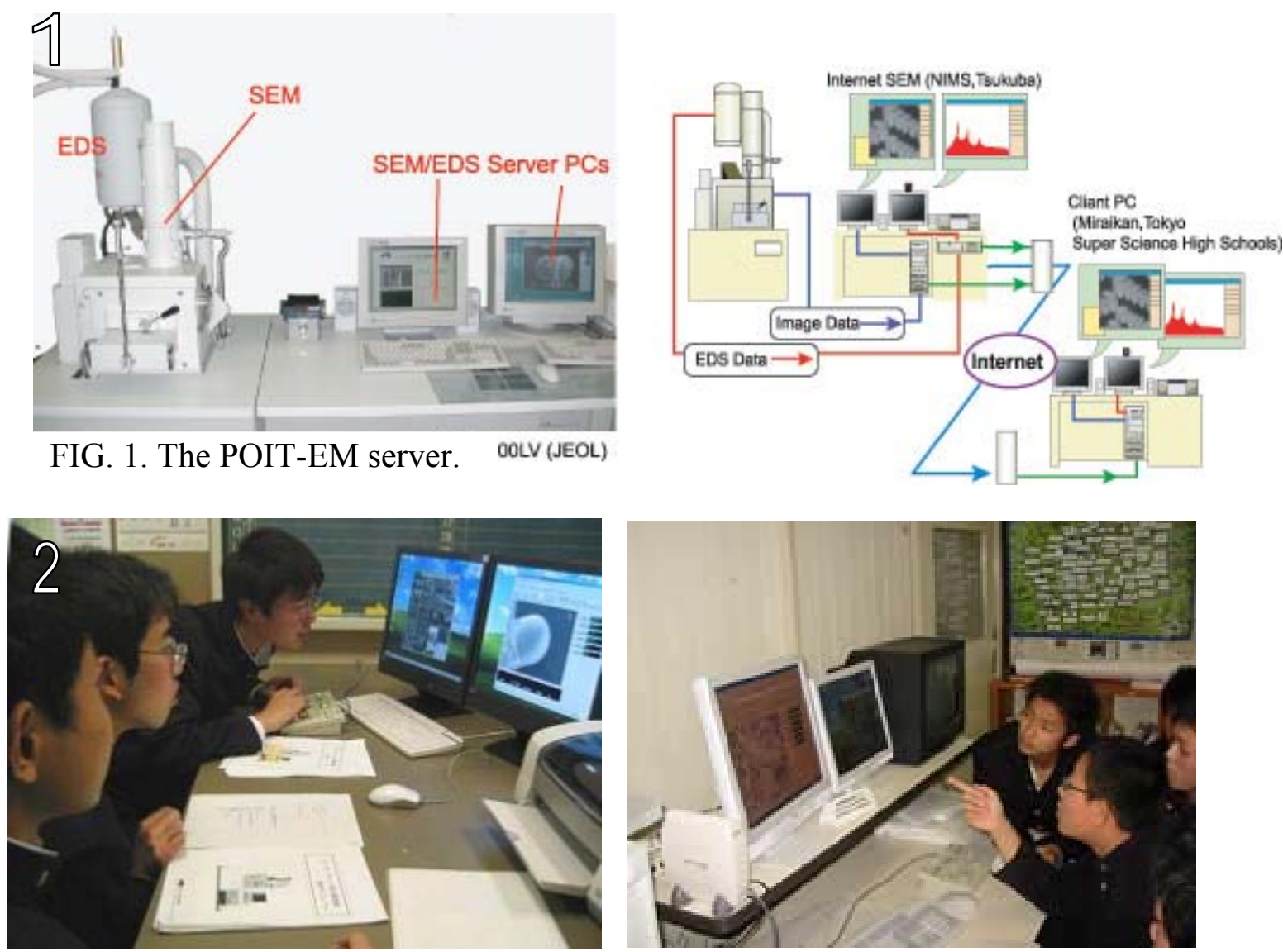

FIG. 2. Client terminals at high schools.

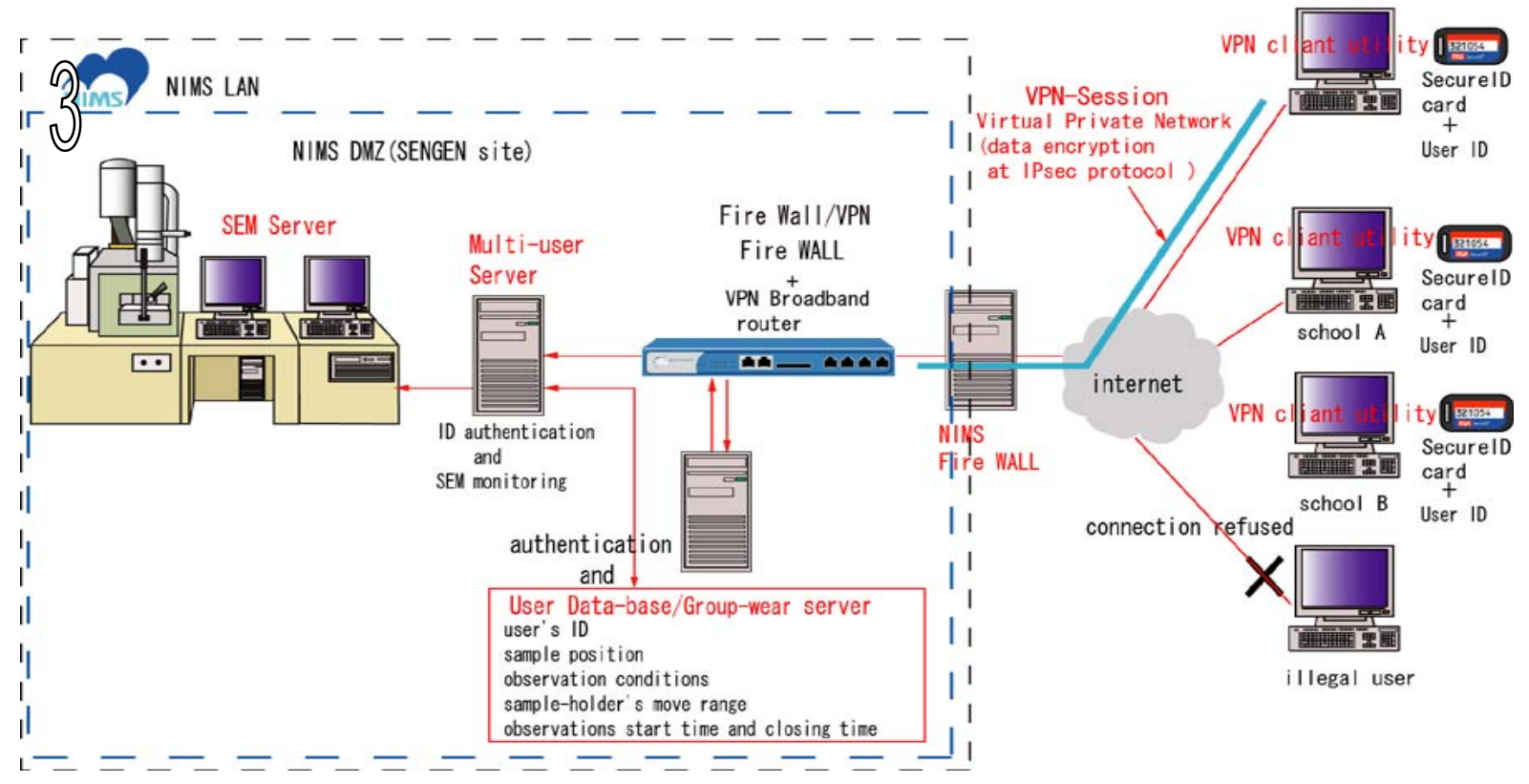

FIG. 3. The multi-users system of POIT-EM (SEM) 